\title{
STABLE MINIMAL SURFACES IN EUCLIDEAN SPACE
}

\author{
MARIO J. MICALLEF
}

To my wife

\section{Introduction}

In this paper we study two-real dimensional minimal surfaces in Euclidean space which are stable, that is, which minimize area on each compact set up to second order. The earliest result for such surfaces is due to S. Bernstein [4], who proved that all complete minimal graphs in $\mathbf{R}^{3}$ (these are automatically stable) are planes. This Bernstein result was generalized by $R$. Osserman in [21] and [22] where he showed that any complete minimal surface in $\mathbf{R}^{3}$ whose Gauss map omits an open set (or even just a set of positive logarithmic capacity) from the sphere must be a plane. In these theorems Osserman makes no assumption about the stability of the minimal surface. The theorem of Osserman was later extended, partially by S. S. Chern [8] and to its full generality by Osserman [23], to minimal surfaces in $\mathbf{R}^{n}$, for any $n$, again without any assumption on stability. F. Xavier [30] has recently strengthened the theorem of Osserman for minimal surfaces in $\mathbf{R}^{3}$ in a remarkable way: he has proved that if the Gauss map of a complete minimal surface in $\mathbf{R}^{3}$ omits 7 or more points from the sphere, then it must be a plane. The relationship of the stable regions on a minimal surface $M$ to the area of their Gaussian image has been studied by J. L. Barbosa and M. doCarmo in [3]. The methods of R. Schoen, L. Simon and S. T. Yau [25] yield a proof of the Bernstein result for stable minimal surfaces $M$ in $\mathbf{R}^{3}$ provided the area growth of a ball of geodesic radius $r$ in $M$ is not larger than $r^{6}$. (This condition is automatically satisfied by all minimal graphs in $\mathbf{R}^{3}$.) A classification theorem for complete stable minimal surfaces in three dimensional manifolds of nonnegative scalar curvature has been obtained by D. Fischer-Colbrie and R. Schoen in [12]. A corollary of their theorem states that all complete oriented stable minimal surfaces in $\mathbf{R}^{3}$ are planes, thereby giving another direct generalization of the Bernstein theorem. This corollary was also proved by M. doCarmo and C. K. Peng [6].

Received April 28, 1983. 
All the results mentioned so far, with the exception of the ones due to Chern [8] and Osserman [23], deal with hypersurfaces. For higher codimensions, the following result due to W. Wirtinger [29] is well known: a holomorphic curve in $\mathbf{C}^{n}\left(=\mathbf{R}^{2 n}\right)$ is absolutely area minimizing with respect to arbitrary compactly supported deformations. Actually, the result as stated is a very special case of a much more general theorem due to $\mathrm{H}$. Federer [11]. A characterization of the Gauss map for holomorphic curves in $\mathbf{C}^{n}$ has been obtained by $\mathrm{H}$. Blaine Lawson, Jr. [18], pp. 164-166.

In view of the Wirtinger result, it is reasonable to ask whether an area minimizing surface in $\mathbf{R}^{n}$ lies in an even dimensional affine subspace of $\mathbf{R}^{n}$ and is holomorphic with respect to some orthogonal complex structure on this even dimensional affine subspace. The result mentioned above, due to D. FischerColbrie and R. Schoen and also due to M. doCarmo and C. K. Peng, shows that this is the case when $n=3$. F. Morgan [20] has shown that the nonzero sum of oriented two-planes in $\mathbf{R}^{n}$ is area minimizing only if all the planes are complex under some complex structure on the span of the planes. In this paper we obtain a partial affirmative answer to the above question for $n \geqslant 4$. The best results are for $n=4$ when, in particular, we prove (Theorem I in §5) that any complete oriented parabolic stable minimal surface in $\mathbf{R}^{4}$ is holomorphic with respect to some orthogonal complex structure on $\mathbf{R}^{4}$. There are plenty of examples of complete parabolic minimal surfaces in Euclidean space: entire two-dimensional minimal graphs (entire means that the graph is defined over all of $\mathbf{R}^{2}$ ), complete minimal surfaces of finite total curvature and also minimal surfaces of quadratic area growth. It is interesting to note that $\mathbf{R}$. Osserman [24, pp. 40-42] has constructed examples of entire minimal graphs in $\mathbf{R}^{4}$ which are not holomorphic with respect to any orthogonal complex structure on $\mathbf{R}^{4}$; these minimal graphs are therefore unstable by our theorem.

Let us now recall that the Grassmannian of oriented two-planes in $\mathbf{R}^{4}$ is homeomorphic to $S^{2} \times S^{2}$. Theorem II concerns stable isometric minimal immersions of an oriented surface with a complete metric into $\mathbf{R}^{4}$ for which one of the projections of the Gauss map onto the factors of $S^{2} \times S^{2}$ omits an open set; the theorem states that such immersions are holomorphic with respect to an orthogonal complex structure on $\mathbf{R}^{4}$. There are many examples of nonholomorphic, complete minimal surfaces in $\mathbf{R}^{4}$ whose Gauss map satisfies the condition required in Theorem II. These must therefore be unstable. In the case that the surface is simply connected, the condition on the Gauss map in Theorem II may be relaxed to one of the projections onto the factors of $S^{2} \times S^{2}$ omitting a set of positive logarithmic capacity.

Following Osserman [24, p. 122] we say that an isometric immersion $F$ : $M^{2} \rightarrow \mathbf{R}^{n}$ of an oriented surface $M$ has a degenerate Gauss map if the Gauss 
image lies in a hyperplane of $\mathbf{C} P^{n-1}$. Thus, the Gauss map of $F$ is degenerate if there exists a fixed, nonzero vector $A \in \mathbf{C}^{n}$ such that $A \cdot F_{z} \equiv 0$, where $z$ is a local complex coordinate on $M$ and $v \cdot w=\Sigma_{i} v_{i} w_{i}$ for $v=\left(v_{1}, \cdots, v_{n}\right), w=$ $\left(w_{1}, \cdots, w_{n}\right) \in \mathbf{C}^{n}$. We also say that the Gauss map of $F$ is $\delta$-degenerate, where $\delta=|A \cdot A| /|A|^{2} \in[0,1]$. Theorem III states that a complete oriented stable minimal surface in $\mathbf{R}^{4}$ whose Gauss image is at least $1 / 3$ degenerate is a plane. It is worth pointing out that surfaces in $\mathbf{R}^{4}$ which lie in a 3-dimensional affine subspace are 1-degenerate. Therefore, Theorem III is a generalization of the theorem that all complete oriented stable minimal surfaces in $\mathbf{R}^{3}$ are planes. At the other extreme, holomorphic curves in $\mathbf{C}^{2}\left(=\mathbf{R}^{4}\right)$ are 0 -degenerate.

Theorem IV provides an affirmative answer to the question addressed in this paper when the surface is oriented, complete, of finite total curvature and genus zero and the ambient Euclidean space is of any dimension.

A first step in proving all these theorems is a theorem (Theorem A in \$3) on a characterization of holomorphic immersions $F: M^{2} \rightarrow \mathrm{C}^{n}$ in terms of a parallel orthogonal splitting of the complexified normal bundle of $M$ into $(1,0)$ and $(0,1)$ subbundles. A complex version of the stability inequality $(\S 2)$ is used to establish the parallel orthogonal splitting required in Theorem A. This complex version of the stability inequality was inspired by a manipulation of Ju. A. Aminov [2]. A similar technique was used by Siu and Yau in [26]. This inequality also suggests that one should look for holomorphic sections of the complexified normal bundle of $M$ of appropriate growth. In $\$ 4$ we use the Gauss map to produce such sections which are used in the proof of Theorem II. In the case of finite total curvature and genus zero (Theorem IV), the Riemann Roch theorem provides the required sections. The proof of Theorem $I$ is somewhat different. The complexified normal bundle of a codimension two oriented submanifold of an oriented Riemannian manifold always splits as the sum of two complex line bundles. In the proof of Theorem I, we utilize the projection of a constant vector field in $\mathbf{C}^{4}$ onto one of these line bundles. When the minimal surface is degenerate (as in Theorem III), each of the projections of $A$ (where $A \cdot F_{z} \equiv 0$ ) onto these line bundles is a (bounded) holomorphic section.

A few words about notation. Covariant differentiation by means of the usual Levi-Civita (Riemannian, flat) connection on the Euclidean space $\mathbf{R}^{n}$ corresponds to the usual directional derivative and so, when it acts on vector-valued functions, we will denote it by $d$. Moreover, we use $\partial_{z}\left(\partial_{\bar{z}}\right)$ instead of $d_{z}\left(d_{\bar{z}}\right)$ when we differentiate vector-valued functions in the direction $\partial / \partial z(\partial / \partial \bar{z})$ along an oriented surface immersed in $\mathbf{R}^{n}$, where $z$ is a local complex coordinate on the surface. Finally, all manifolds are assumed to be $C^{\infty}$ and connected. 
The paper is organized as follows:

$\S 2$ contains the complex version of the stability inequality. In $\$ 3$ we describe a characterization of holomorphic immersions $F: M^{2} \rightarrow \mathrm{C}^{n}$ (Theorem A) and its relation to the stability inequality. In $\$ 4$ we construct meromorphic sections of the complexified normal bundle of a minimal surface in $\mathbf{R}^{4}$ by means of the Gauss map. $\$ 5$ contains the main theorems in this paper.

The results in this paper are based on the author's doctoral dissertation under the direction of Professor Richard M. Schoen. It is a pleasure to express my gratitude to him for his inspiring guidance, patience and encouragement and also for his contribution to some parts of the proof of Theorem IV (the higher dimensional case).

\section{A complex version of the stability inequality}

The condition that a minimal submanifold $M$ of the Euclidean space $\mathbf{R}^{n}$ be stable is expressed by the following inequality (see, for example, [18]) that has to hold for all compactly supported normal sections $s$ (a normal section is a section of the normal bundle $N M$ of $M$ ):

$$
\int_{M}\left|(d s)^{T}\right|^{2} \leqslant \int_{M}\left|(d s)^{N}\right|^{2}
$$

In (2.1), $d s=\left(d s_{1}, \cdots, d s_{n}\right)$, where $s=\left(s_{1}, \cdots, s_{n}\right)$, the superscripts $T$ and $N$ denote orthogonal projection onto the tangent space and normal space of $M$ respectively, $|d s|$ is the length of the vector-valued one-form $d s$ with respect to the induced metric on $M$ (similarly for $\left|(d s)^{T}\right|$ and $\left.\left|(d s)^{N}\right|\right)$ and the integrations are carried out with respect to the volume element for the induced metric on $M$. Inequality (2.1) is still valid for compactly supported sections of $N_{\mathrm{C}} M=N M \otimes_{\mathrm{R}} \mathrm{C}$, the complexified normal bundle of $M$; all we have to do is simply add up the stability inequalities for the real and imaginary parts of $s$. From now on we assume $M$ to be a two-real dimensional oriented surface. Then, isothermal parameters for the induced metric together with the orientation on the surface give rise to a complex structure on $M$. Let $z$ denote a complex coordinate for this complex structure. We can then write $d s=\partial s+\bar{\partial} s$ where $\partial s=\left(\partial_{z} s\right) d z$ and $\bar{\partial} s=\left(\partial_{\bar{z}} s\right) d \bar{z}$. Thus (2.1) may be rewritten in the following way:

$$
2 \int_{M}\left|(d s)^{T}\right|^{2} \leqslant \int_{M}|d s|^{2}=\int_{M}|\partial s|^{2}+\int_{M}|\bar{\partial} s|^{2} .
$$


Integration by parts yields $\int_{M}|\partial s|^{2}=\int_{M}|\bar{\partial} s|^{2}$ because $\mathbf{R}^{n}$ is flat and $s$ has compact support. Therefore

$$
2 \int_{M}\left|(\partial s)^{T}\right|^{2}+2 \int_{M}\left|(\bar{\partial} s)^{T}\right|^{2} \leqslant 2 \int_{M}|\partial s|^{2}
$$

that is

$$
\int_{M}\left|(\partial s)^{T}\right|^{2} \leqslant \int_{M}\left|(\bar{\partial} s)^{N}\right|^{2}
$$

Similarly,

$$
\int_{M}\left|(\bar{\partial} s)^{T}\right|^{2} \leqslant \int_{M}\left|(\partial s)^{N}\right|^{2}
$$

It is perhaps worth noting that (2.1), (2.2) and (2.3) are all equivalent to one another.

As was mentioned in the introduction, the above manipulation was inspired by a manipulation due to Ju. A. Aminov in [2]. He adds the stability inequalities for two orthogonal normal variations of equal length, mixes terms and integrates by parts to obtain an inequality which can be derived from (2.2). Our manipulation is more general in that the real and imaginary parts of $s$ are required to be neither orthogonal nor of equal length.

The usefulness of inequalities (2.2) and (2.3) will become apparent in the next section.

\section{A characterization of holomorphic immersions $F: M^{2 m} \rightarrow \mathrm{C}^{n}$}

\section{and its relation to stability}

Let $N^{2 n}$ be a Kähler manifold (of real dimension $2 n$ ). We denote the complex structure and Levi-Civita (Riemannian) connection on $T N$ by $J$ and $\nabla$ respectively. By extending $J$ to $T_{\mathbf{C}} N=T N \otimes_{\mathrm{R}} \mathrm{C}$, the complexified tangent bundle of $N$, we have $T_{\mathbf{C}} N=T_{\mathbf{C}} N^{1,0} \oplus T_{\mathbf{C}} N^{0,1}$, where $T_{\mathbf{C}} N^{1,0}$ and $T_{\mathbf{C}} N^{0,1}$ are the eigenspaces of $J$ corresponding to the eigenvalues $+i$ and $-i$ respectively. Since $N$ is Kähler, this splitting of $T_{\mathbf{C}} N$ into $(1,0)$ and $(0,1)$ subbundles is preserved by $\nabla$. Moreover $s \cdot t \equiv 0$ for all sections $s, t$ of $T_{\mathrm{C}} N^{1,0}$, where $s \cdot t$ is the inner product of $s$ and $t$ taken with respect to the Riemannian (indeed, Hermitian) metric on $T N$ extended complex linearly in both arguments to $T_{\mathrm{C}} N$. In other words $T_{\mathrm{C}} N^{1,0}$ is orthogonal to $T_{\mathrm{C}} N^{0,1}$ with respect to the inner product $\langle\cdot, \cdot\rangle$ which is defined by $\langle s, t\rangle=s \cdot \bar{t}$, where $\bar{t}$ is the complex conjugate of $t$. (Here we are using the fact that $T_{\mathbf{C}} N^{0,1} \cong \overline{T_{\mathbf{C}} N^{1,0}}$.) 
If $F: M^{2 m} \rightarrow N^{2 n}$ is a holomorphic immersion of a complex manifold $M^{2 m}$ (of real dimension $2 \mathrm{~m}$ ) into $N$, then it is easy to verify from the above comments that $M^{2 m}$ itself becomes a Kähler manifold when equipped with the induced metric and that:

(i) $T_{\mathbf{C}} M \cong T_{\mathbf{C}} M^{1,0} \oplus \overline{T_{\mathbf{C}} M^{1,0}}, N_{\mathbf{C}} M \cong N_{\mathbf{C}} M^{1,0} \oplus \overline{N_{\mathbf{C}} M^{1,0}}$

(ii) $T_{\mathbf{C}} M^{1,0} \oplus N_{\mathbf{C}} M^{1,0}$ is orthogonal to $\overline{T_{\mathbf{C}} M^{1,0}} \oplus \overline{N_{\mathbf{C}} M^{1,0}}$ with respect to $\langle\cdot, \cdot\rangle$.

(iii) $\nabla: \Gamma\left(T_{\mathbf{C}} M^{1,0} \oplus N_{\mathbf{C}} M^{1,0}\right) \rightarrow \Gamma\left(\left(T_{\mathbf{C}} M^{1,0} \oplus N_{\mathbf{C}} M^{1,0}\right) \otimes T^{*} M\right)$.

In the above, we are still denoting by $\nabla$ the induced connection on $F^{*}\left(T_{\mathrm{C}} N\right)$. The converse of the above observation is not true in general. However, we do have the following

Theorem A. Let $F: M^{2 m} \rightarrow \mathbf{R}^{2 n}$ be an immersion, with $\mathbf{R}^{2 n}$ having the usual Euclidean metric. Suppose that there exist vector bundles $E$ and $V$ over $M$ which satisfy conditions (i), (ii) and (iii) below:

(i) $T_{\mathbf{C}} M \cong E \oplus \bar{E}, N_{\mathbf{C}} M \cong V \oplus \bar{V}$.

(ii) $E \oplus V$ is orthogonal to $\bar{E} \oplus \bar{V}$ with respect to $\langle\cdot, \cdot\rangle$.

(iii) $d: \Gamma(E \oplus V) \rightarrow \Gamma\left((E \oplus V) \otimes T^{*} M\right)$.

Then there exist complex structures $\tilde{J}$ and $J$ on $M^{2 m}$ and $\mathbf{R}^{2 n}$ respectively such that $\tilde{J}$ is orthogonal with respect to the metric induced on $M$ by the immersion, $J$ is orthogonal with respect to the Euclidean inner product on $\mathbf{R}^{2 n}$ and $F$ is holomorphic with respect to $\tilde{J}$ and $J$.

Remark. $J$ is actually constant on all of $\mathbf{R}^{2 n}$, thereby giving $\mathbf{R}^{2 n}$ the usual Kähler structure of $\mathbf{C}^{n}$. Since $F$ is holomorphic, $\tilde{J}$ and the induced metric also yield a Kähler structure on $M$.

Proof of Theorem A. Let $\left\{e_{1}, \cdots, e_{m}\right\}$ and $\left\{e_{m+1}, \cdots, e_{n}\right\}$ be local frame fields for $E$ and $V$ respectively which are orthonormal with respect to $\langle\cdot, \cdot\rangle$. By assumption (iii),

$$
d e_{A}=\sum_{B} \omega_{A B} \otimes e_{B}
$$

for some $n \times n$ matrix of one-forms $\left(\omega_{A B}\right), A, B \in\{1, \cdots, n\}$. We define a $2 n \times 2 n$ complex matrix $C$ by ${ }^{t} C=\left(e_{1}, \cdots, e_{n}, \bar{e}_{1}, \cdots, \bar{e}_{n}\right)$, where the superscript $t$ denotes transpose so that $C$ is the matrix whose rows are the $2 n$-tuples of complex numbers with which the $e_{A}$ and $\bar{e}_{A}$ are being identified by means of the canonical coordinate functions on $\mathbf{R}^{2 n}$. From $(*)$ we have $d C=\omega \otimes C$, where

$$
\omega=\left(\begin{array}{cc}
\left(\omega_{A B}\right) & 0 \\
0 & \left(\bar{\omega}_{A B}\right)
\end{array}\right)
$$


Let

$$
J_{0}=\left(\begin{array}{rrrrr}
-i & & & & 0 \\
& \ddots & & & \\
& & -i & & \\
& & & \ddots & \\
0 & & & & +i
\end{array} \mid,\right.
$$

where $i$ and $-i$ each appear $n$-times along the diagonal. Define $J$ by $J=C^{-1} J_{0} C$. Then $J^{2}=C^{-1}\left(J_{0}\right)^{2} C=-1_{2 n}$, the negative of the identity matrix on $\mathbf{C}^{2 n}$. Moreover $J$ is a matrix with real entries: by assumption (ii),

$$
C^{t} C=\left(\begin{array}{cc}
0 & 1_{n} \\
1_{n} & 0
\end{array}\right),
$$

where $1_{n}$ is the identity matrix on $\mathbf{C}^{n}$ and $C^{-1}={ }^{t} C$. Therefore,

$$
J-\bar{J}=\overline{{ }^{t} C} J_{0} C+{ }^{t} C J_{0} \bar{C}=\overline{{ }^{t} C}\left(J_{0} C{ }^{t} C+C{ }^{t} C J_{0}\right) \bar{C}=0 .
$$

Finally,

$$
d J=-C^{-1} d C C^{-1} J_{0} C+C^{-1} J_{0} d C=-C^{-1}\left(\omega J_{0}-J_{0} \omega\right) C=0 .
$$

Therefore $J$ is constant along $F(M)$. Thus, we can define a complex structure on $\mathbf{R}^{2 n}$ by declaring it to be $J$ at any point; this is possible because $J$ is constant along $F(M)$ and $J$ has real entries. $J$ is orthogonal with respect to the Euclidean inner product because it was defined by means of an orthonormal frame. Note that $J$ preserves $E$ (it multiplies sections of it by $i$ ), that is, $J$ preserves $F_{*}\left(T_{p} M\right)$ at all points $F(p)$ in $F(M)$. But this is precisely the definition for $M$ to be a complex submanifold of $\mathbf{R}^{2 n}$ with respect to the complex structure $J$. (The complex structure $\tilde{J}$ on $T M$ is, of course, defined by demanding that $F$ be holomorphic with respect to $\tilde{J}$ and $J$, that is, $\tilde{J}$ is defined by $\tilde{J}=F_{*}^{-1} \circ J \circ F_{*}$.) The proof of Theorem A is complete.

Remark. The above proof also works for immersions $F: M^{2 m} \rightarrow T^{2 n}$, where $T^{2 n}=\mathbf{R}^{2 n} / \mathbf{Z}^{2 n}$ is the flat $2 n$-real dimensional torus. (This is because the tangent bundle of $T^{2 n}$, like that of $\mathbf{R}^{2 n}$, admits a global parallel orthonormal frame.) Therefore Theorem $A$ is valid for such immersions too.

We now restrict our attention to $m=1$ and $n=2$, that is, to immersions $F$ : $M^{2} \rightarrow \mathbf{R}^{4}$ of an oriented surface $M$ into Euclidean 4-space, and discuss the relation of Theorem $\mathrm{A}$ in this special case to the stability inequality (2.2). Let $\left\{e_{1}, e_{2}, e_{3}, e_{4}\right\}$ be a local oriented orthonormal frame for $\mathbf{R}^{4}$ over an open set of $F(M)$ such that $\left\{e_{1}, e_{2}\right\}$ and $\left\{e_{3}, e_{4}\right\}$ are local oriented orthonormal frames for the tangent and normal bundles of $M$ respectively. In this case, therefore, 
$T M$ and $N M$ can each be given a complex structure, namely, rotation by $90^{\circ}$ in an anticlockwise direction. Let $E=\left(T_{\mathbf{C}} M\right)^{1,0}$ and $V=\left(N_{\mathbf{C}} M\right)^{1,0}$, where type $(1,0)$ is meant with respect to the complex structure just mentioned. The fibres of $E$ are then locally spanned by $\left(e_{1}-i e_{2}\right)$ and those of $V$ by $\left(e_{3}-i e_{4}\right) . E$ and $V$ satisfy conditions (i) and (ii) required in the hypotheses of Theorem A. Moreover,

$$
d\left(e_{1}-i e_{2}\right)=i \omega_{12} \otimes\left(e_{1}-i e_{2}\right)+\left[d\left(e_{1}-i e_{2}\right)\right]^{N}
$$

and

$$
d\left(e_{3}-i e_{4}\right)=i \omega_{34} \otimes\left(e_{3}-i e_{4}\right)+\left[d\left(e_{3}-i e_{4}\right)\right]^{T},
$$

where $\omega_{12}$ and $\omega_{34}$ are the connection one-forms for $T M$ and $N M$ respectively. Therefore assumption (iii) of Theorem $\mathrm{A}$ is satisfied if and only if

$$
\left[d\left(e_{3}-i e_{4}\right)\right] \cdot\left(e_{1}-i e_{2}\right) \equiv 0 \text {. }
$$

Let $z$ be a local complex coordinate on $M$. Then $F_{z}=\mu\left(e_{1}-i e_{2}\right)$, where $\mu$ is a locally defined nonvanishing complex valued function. Thus we see that $(* *)$ is satisfied if and only if

$$
\partial\left(e_{3}-i e_{4}\right) \cdot F_{z} \equiv 0 \text { and } \bar{\partial}\left(e_{3}-i e_{4}\right) \cdot F_{z} \equiv 0,
$$

where we have used $d\left(e_{3}-i e_{4}\right)=\partial\left(e_{3}-i e_{4}\right)+\bar{\partial}\left(e_{3}-i e_{4}\right)$. But

$$
\begin{aligned}
\bar{\partial}\left(e_{3}-i e_{4}\right) \cdot F_{z} & =\left[\partial_{\bar{z}}\left(e_{3}-i e_{4}\right) \cdot F_{z}\right] d \bar{z} \\
& =-\left[\left(e_{3}-i e_{4}\right) \cdot F_{z \bar{z}}\right] d \bar{z} \\
& =\left[\partial_{z}\left(e_{3}-i e_{4}\right) \cdot F_{\bar{z}}\right] d \bar{z}
\end{aligned}
$$

Therefore $(* * *)$ is equivalent to $\partial_{z}\left(e_{3}-i e_{4}\right) \cdot F_{z} \equiv 0$ and $\partial_{z}\left(e_{3}-i e_{4}\right) \cdot F_{\bar{z}} \equiv 0$, that is, to $\left[\partial\left(e_{3}-i e_{4}\right)\right]^{T} \equiv 0$. But if $s$ is a section of $V$, that is, locally $s=g\left(e_{3}-i e_{4}\right)$, then $(\partial s)^{T}=g\left[\partial\left(e_{3}-i e_{4}\right)\right]^{T}$. This implies that, for an immersion of an oriented two-real dimensional surface $M$ into $\mathbf{R}^{4}$ to be holomorphic, all that has to be true is that on the neighbourhood of any point in $M$, there exists a local nonzero section $s$ of $V$ with the property that $(\partial s)^{T}=0$. But $(\partial s)^{T}$ is precisely the term on the left in the stability inequality (2.2). This is why there is some hope of being able to use the complex version of the stability inequality derived in $\$ 2$ to solve the problem addressed in this paper.

Remark. A. N. Wang in [28] has already noticed that if $(\partial s)^{T}=0$ for all sections $s$ of $V$, then $F: M^{2} \rightarrow R^{4}$ is holomorphic with respect to some orthogonal complex structure on $\mathbf{R}^{4}$. He states the condition that $(\partial s)^{T}=0$ in terms of the second fundamental form. 


\section{Construction of meromorphic sections of the normal bundle}

\section{of a minimal surface in $R^{4}$ by means of the Gauss map}

For more details than will be given in this section we refer the reader to Hoffman and Osserman [14], Lawson [18] and the author's doctoral dissertation [19].

Let $G_{k, n}$ denote the Grassmannian of oriented $k$-planes in $\mathbf{R}^{n}$. Let $F$ : $M^{k} \rightarrow \mathbf{R}^{n}$ be an immersion of a $k$-real dimensional oriented manifold into the Euclidean space $\mathbf{R}^{n}, 2 \leqslant k \leqslant n-1$. The generalized Gauss map $G: M^{k} \rightarrow G_{k, n}$ is defined by $G(p)=F_{*}\left(T_{p} M\right)$, where $T_{p} M$ is the oriented tangent space of $M$ at $p$ and $F_{*}\left(T_{p} M\right)$ is translated from $F(p)$ to the origin of $\mathbf{R}^{n}$.

We now focus on the special case $k=2$ and recall that $G_{2, n}$ may be identified with the quadric $Q_{n-2} \subset \mathbf{C} P^{n-1}$ defined by $w \cdot w=\sum_{j}\left(w_{j}\right)^{2}=0$ where $w=\left(w_{1}, \cdots, w_{n}\right)$ is a homogeneous coordinate for a point in $\mathbf{C} P^{n-1}$. If $z$ is a local complex coordinate on $M^{2}$, then $F_{z}(p)$ is a homogeneous coordinate for $G(p)$.

From now on, we further restrict our attention to $n=4$ and recall that $Q_{2}$ (and therefore $G_{2,4}$ ) may be identified with $S^{2} \times S^{2}$. Let $P$ and $Q$ be two fixed vectors in $\mathbf{C}^{4}$ such that $P \cdot P=P \cdot Q=P \cdot \bar{Q}=Q \cdot Q=0$ and $P \cdot \bar{P}=Q \cdot$ $\bar{Q}=1$. (This makes $\{P, \bar{P}, Q, \bar{Q}\}$ an orthonormal basis for $\mathbf{C}^{4}$.) If $F: M^{2} \rightarrow \mathbf{R}^{4}$ is an immersion then

$$
F_{z}=F_{z} \cdot P\left(\bar{P}-\omega_{1} \omega_{2} P+\omega_{1} \bar{Q}+\omega_{2} Q\right)
$$

outside the zero set of $F_{z} \cdot P$, where

$$
\omega_{1}=\frac{F_{z} \cdot Q}{F_{z} \cdot P}, \omega_{2}=\frac{F_{z} \cdot \bar{Q}}{F_{z} \cdot P} .
$$

The significance of $\omega_{1}$ and $\omega_{2}$ is that $\left(\omega_{1}(p), \omega_{2}(p)\right)$ is the coordinate of $G(p)$ under stereographic projection of the two factors of $S^{2} \times S^{2}$ from $\infty_{1}$ and $\infty_{2}$ respectively, where $\left(\infty_{1}, \infty_{2}\right)$ is the point in $S^{2} \times S^{2}\left(=Q_{2} \subset \mathbf{C} P^{3}\right)$ with $P$ as a homogeneous coordinate. Thus

$$
\bar{P}-\omega_{1} \omega_{2} P+\omega_{1} \bar{Q}+\omega_{2} Q
$$

is a section of the holomorphic tangent bundle of $M$ (outside the zero set of $F_{z} \cdot P$ ) written in terms of the Gauss map of $F$. Indeed, this section is none other than $F_{z} /\left(F_{z} \cdot P\right)$.

Let $\Pi$ be a given oriented two-plane in $\mathbf{R}^{4}$ and let $\Pi^{\prime}$ be the two-plane normal to $\Pi$, with the orientation on $\Pi^{\prime}$ being chosen as follows: if $\left\{e_{1}, e_{2}\right\}$ and $\left\{e_{3}, e_{4}\right\}$ are bases for $\Pi$ and $\Pi^{\prime}$ respectively such that $e_{1} \wedge e_{2}$ and $e_{1} \wedge e_{2} \wedge e_{3} \wedge e_{4}$ determine the orientations on $\Pi$ and $\mathbf{R}^{4}$, then the orientation on $\Pi^{\prime}$ is that determined by $e_{3} \wedge e_{4}$. It turns out that, regarding $\Pi$ and $\Pi^{\prime}$ as 
points of $S^{2} \times S^{2}, \Pi^{\prime}$ is obtained from $\Pi$ by the antipodal map on the second factor. This fact was observed by Chern and Spanier [10] and a different proof was given by the author in [19]. Using this fact it is clear that, on replacing $\omega_{2}$ in (4.2) by $-1 / \bar{\omega}_{2}$ and then multiplying throughout by $\bar{\omega}_{2}$, the resulting expression will be a section of $N_{\mathrm{C}} M$ outside the zero set of $F_{z} \cdot P$. Indeed, if we write

$$
e_{3}-i e_{4}=\sqrt{2} \frac{\left\{\omega_{2} \bar{P}+\omega_{1} P+\omega_{1} \bar{\omega}_{2} \bar{Q}-Q\right\}}{\left(1+\left|\omega_{1}\right|^{2}\right)^{1 / 2}\left(1+\left|\omega_{2}\right|^{2}\right)^{1 / 2}},
$$

then $\left\{e_{3}, e_{4}\right\}$ is an oriented orthonormal frame for $N_{\mathbf{C}} M$ outside the zero set of $F_{z} \cdot P$. This fact was also observed by Hoffman and Osserman in [15].

Let $D$ denote covariant differentiation along $M$ in the complexified normal bundle. Then $D\left(e_{3}-i e_{4}\right)=i \omega_{34} \otimes\left(e_{3}-i e_{4}\right)$, where $\omega_{34}$ is the connection one-form which defines $D$. The computation of $\omega_{34}$ is easy:

$$
\begin{aligned}
\omega_{34} & =-\frac{i}{2}\left[D\left(e_{3}-i e_{4}\right)\right] \cdot\left(e_{3}+i e_{4}\right) \\
& =-\frac{i}{2}\left[d\left(e_{3}-i e_{4}\right)\right] \cdot\left(e_{3}+i e_{4}\right) \\
& =\frac{i}{2}\left(\frac{\omega_{1} d \bar{\omega}_{1}-\bar{\omega}_{1} d \omega_{1}}{1+\left|\omega_{1}\right|^{2}}-\frac{\omega_{2} d \bar{\omega}_{2}-\bar{\omega}_{2} d \omega_{2}}{1+\left|\omega_{2}\right|^{2}}\right) .
\end{aligned}
$$

We now restrict ourselves to considering only minimal immersions $F$ : $M^{2} \rightarrow \mathbf{R}^{4}$ and under this assumption we look for complex valued normal sections $s$ for which $D_{\bar{z}} s=0$. We recall that the minimality of $F$ implies that $F$ is harmonic and therefore that $\omega_{1}$ and $\omega_{2}$ are meromorphic. Thus (4.4) simplifies to

$$
\omega_{34}=\frac{i}{2}\left(\bar{\partial} \log \frac{1+\left|\omega_{1}\right|^{2}}{1+\left|\omega_{2}\right|^{2}}-\partial \log \frac{1+\left|\omega_{1}\right|^{2}}{1+\left|\omega_{2}\right|^{2}}\right) .
$$

We look for sections $s$ of the form $g\left(e_{3}-i e_{4}\right)$, where $g$ is a smooth complex valued function. $D_{\bar{z}} s=0$ implies $\left\{\partial_{\bar{z}} g+i g \omega_{34}\left(\partial_{\bar{z}}\right)\right\}\left(e_{3}-i e_{4}\right)=0$. Using (4.5) it is easily seen that

$$
g=\left(\frac{1+\left|\omega_{1}\right|^{2}}{1+\left|\omega_{2}\right|^{2}}\right)^{1 / 2} h
$$

where $h$ is any holomorphic function.

At this point we recall a proposition due to Koszul and Malgrange [17]: Let $V$ be a complex vector bundle over a Riemann surface $M$ and suppose that $V$ has a connection defined on it. Then $V$ can be made into a holomorphic vector 
bundle over $M$ with $\bar{\partial}$ operator equal to the $(0,1)$ part of its connection, that is, a section $s$ of $V$ is holomorphic if and only if $D_{\bar{z}} s=0$, where $z$ is a local complex coordinate on $M$ and $D$ is covariant differentiation in $V$ defined by means of the connection on $V$.

Thus we see that what we have done two paragraphs above is to use the Gauss map to construct sections of $V$, the complex line subbundle of $N_{\mathbf{C}} M$ which was introduced in $\$ 3$ after the proof of Theorem $A$ and whose fibres are spanned by $e_{3}-i e_{4}$, which are meromorphic with respect to the holomorphic structure on $V$ given by the above proposition of Koszul and Malgrange, in the case when the immersion $F: M^{2} \rightarrow \mathbf{R}^{4}$ is minimal. We may obtain meromorphic sections of $\bar{V}$ (whose fibres are spanned by $e_{3}+i e_{4}$ and which is equipped with the holomorphic structure given by the proposition of Koszul and Malgrange) by noticing that if $s$ is a meromorphic section of $V$, then, $\bar{s} /|s|^{2}$ is a meromorphic section of $\bar{V}$. This can be seen as follows: outside the zeros and poles of $s, D_{z} s=f s$ for some complex valued function $f$. To compute $f$ in terms of $s$ we note that $\partial_{z}(s \cdot \bar{s})=f(s \cdot \bar{s})$ and therefore, $f=\partial_{z} \log |s|^{2}$. Thus

$$
D_{\bar{z}}\left(\frac{\bar{s}}{|s|^{2}}\right)=\left\{\partial_{\bar{z}} \frac{1}{|s|^{2}}+\frac{1}{|s|^{2}} \bar{f}\right\} \bar{s}=0 .
$$

The zeros and poles of $s$ can be easily handled by the reader and we regard the observation as proved. For future reference we record here the results of this section:

$$
s=\frac{1}{\sqrt{2}}\left(\frac{1+\left|\omega_{1}\right|^{2}}{1+\left|\omega_{2}\right|^{2}}\right)^{1 / 2}\left(e_{3}-i e_{4}\right)
$$

is a meromorphic section of $V$ where $\left(e_{3}-i e_{4}\right)$ is given by (4.3),

$$
\begin{gathered}
|s|^{2}=\frac{1+\left|\omega_{1}\right|^{2}}{1+\left|\omega_{2}\right|^{2}}, \\
\frac{\bar{s}}{|s|^{2}}=\frac{1}{\sqrt{2}}\left(\frac{1+\left|\omega_{2}\right|^{2}}{1+\left|\omega_{1}\right|^{2}}\right)^{1 / 2}\left(e_{3}+i e_{4}\right)
\end{gathered}
$$

is a meromorphic section of $\bar{V}$,

$$
\left|\frac{\bar{s}}{|s|^{2}}\right|^{2}=\frac{1+\left|\omega_{2}\right|^{2}}{1+\left|\omega_{1}\right|^{2}} .
$$




\section{The main results}

Throughout this section $M$ will denote an oriented surface (two-real dimensional manifold) with a metric. The complex structure on $M$ shall always be that defined by the orientation and isothermal parameters for the metric. Thus, if $z$ is a local complex coordinate, $z=x+i y$, where $(x, y)$ are oriented isothermal parameters for the metric. We recall that a Riemann surface is said to be parabolic if it does not admit positive nonconstant superharmonic functions.

Theorem I. Let $F: M^{2} \rightarrow \mathbf{R}^{4}$ be an isometric stable minimal immersion of a complete oriented parabolic surface into Euclidean 4-space. Then $F$ is holomorphic with respect to some orthogonal complex structure on $\mathbf{R}^{4}$.

Before giving the proof of Theorem I we point out some corollaries and make some remarks. In these comments we economize on words by referring to an immersion which is holomorphic with respect to some orthogonal complex structure on $\mathbf{R}^{4}$ simply as being holomorphic.

Corollary 5.1. A complete stable minimal surface in $\mathbf{R}^{4}$ which is an entire graph is holomorphic.

Proof. A corollary to a theorem of Osserman [24, pp. 37-40] states that a minimal surface in Euclidean space which is an entire graph is conformally equivalent to the complex plane. In particular, it is parabolic and Corollary 5.1 is an immediate consequence of Theorem $\mathrm{I}$.

Remark. The assumption of stability cannot be dropped from Corollary 5.1. Osserman [24, pp. 40-42] has constructed examples of entire two-dimensional minimal graphs in $\mathbf{R}^{4}$ which are not holomorphic with respect to any orthogonal complex structure on $\mathbf{R}^{4}$. These graphs are therefore unstable by Corollary 5.1 .

Corollary 5.2. A complete oriented stable minimal surface in $\mathbf{R}^{4}$ which is of finite total curvature is holomorphic.

Proof. A theorem of Chern and Osserman [9] states that a minimal surface of finite total curvature in Euclidean space is conformally equivalent to a compact Riemann surface with finitely many punctures. In particular, such surfaces are parabolic and this corollary is again an immediate consequence of Theorem 1.

Corollary 5.3. A complete stable oriented minimal surface in $\mathbf{R}^{4}$ which has quadratic area growth is holomorphic.

Proof. A complete surface of quadratic area growth is parabolic by a theorem of Cheng and Yau [7].

We now prove some lemmas that will be needed for the proof of Theorem I. We recall an observation made in the paragraph following the proof of 
Theorem A in §3: for an immersion $F: M^{2} \rightarrow \mathbf{R}^{4}, N_{\mathbf{C}} M \cong V \oplus \bar{V}$. If $v$ is any vector in $\mathrm{C}^{4}$, let $v^{1,0}$ and $v^{0,1}$ denote the orthogonal projection of $v$ onto $V$ and $\bar{V}$ respectively. We let $D$ denote covariant differentiation in $N_{\mathbf{C}} M$ and $z=x+$ iy will be a local complex coordinate. Finally, if $v=\left(v_{1}, \cdots, v_{n}\right)$ and $w=$ $\left(w_{1}, \cdots, w_{n}\right)$ are two vectors in $\mathrm{C}^{n}$, then $v \cdot w=\Sigma_{j} v_{j} w_{j}$.

Lemma 5.1. For a minimal immersion $F: M^{2} \rightarrow \mathbf{R}^{4},\left(F_{z z}\right)^{1,0} \otimes(d z)^{2}$ is a holomorphic quadratic differential with values in $V$ and $\left(F_{z z}\right)^{0,1} \otimes(d z)^{2}$ is a holomorphic quadratic differential with values in $\bar{V}$. (Here, holomorphic is meant with respect to the holomorphic structure given by the proposition of Koszul and Malgrange mentioned in \$4.)

Proof. Since $D$ preserves $V$ and $\bar{V}$, all we have to check is that $D_{\bar{z}}\left(F_{z z}\right)^{N}=0$. Keeping in mind that $F_{z} \cdot F_{z}=0$ by definition of isothermal parameters and therefore that $F_{z} \cdot F_{z z}=0$ we compute:

$$
\begin{aligned}
\left(F_{z z}\right)^{N} & =F_{z z}-\left(F_{z z}\right)^{T} \\
& =F_{z z}-\frac{1}{\left|F_{z}\right|^{2}}\left(F_{z z} \cdot F_{z}\right) F_{\bar{z}}-\frac{1}{\left|F_{z}\right|^{2}}\left(F_{z z} \cdot F_{\bar{z}}\right) F_{z} \\
& =F_{z z}-\frac{1}{\left|F_{z}\right|^{2}}\left(F_{z z} \cdot F_{\bar{z}}\right) F_{z} .
\end{aligned}
$$

Therefore

$$
D_{\bar{z}}\left(F_{z z}\right)^{N}=\left\{\partial_{\bar{z}}\left(F_{z z}-\frac{1}{\left|F_{z}\right|^{2}}\left(F_{z z} \cdot F_{\bar{z}}\right) F_{z}\right)\right\}^{N}=0
$$

by minimality. The proof of the lemma is complete.

Corollary 5.4. For a minimal immersion $F: M^{2} \rightarrow \mathbf{R}^{4}$, each of $\left|\left(F_{z z}\right)^{1,0}\right|$ and $\left|\left(F_{z z}\right)^{0,1}\right|$ either vanishes identically or only at isolated points.

Note that the statement of Corollary 5.4 is independent of the choice of the local complex coordinate $z$.

Lemma 5.2. If $F: M^{2} \rightarrow \mathbf{R}^{4}$ is an isometric minimal immersion and $a \in \mathbf{C}^{4}$ is a fixed vector then

$$
\begin{aligned}
D_{z} D_{\bar{z}} a^{1,0}= & -\frac{1}{\left|F_{z}\right|^{2}}\left(F_{z z} \cdot a^{1,0}\right)\left(F_{\bar{z} \bar{z}}\right)^{1,0} \\
& -\frac{1}{\left|F_{z}\right|^{2}}\left(\left(F_{z z}\right)^{1,0} \cdot a\right)\left(F_{\bar{z} \bar{z}}\right)^{1,0} .
\end{aligned}
$$

Proof. Let $\left\{e_{3}, e_{4}\right\}$ be a local oriented orthonormal frame for $N_{\mathrm{C}} M$ and let $\varepsilon=\left(e_{3}-i e_{4}\right) / \sqrt{2}$. Then

$$
a^{1,0}=(a \cdot \bar{\varepsilon}) \varepsilon
$$


Therefore

$$
\begin{aligned}
D_{\bar{z}} a^{1,0} & =\left\{a \cdot\left(\partial_{\bar{z}} \bar{\varepsilon}\right)^{T}\right\} \varepsilon+\left\{a \cdot\left(-i \omega_{34}\left(\partial_{\bar{z}}\right) \bar{\varepsilon}\right)\right\} \varepsilon+(a \cdot \bar{\varepsilon}) i \omega_{34}\left(\partial_{\bar{z}}\right) \varepsilon \\
& =\left\{a \cdot\left(\partial_{\bar{z}} \bar{\varepsilon}\right)^{T}\right\} \varepsilon,
\end{aligned}
$$

where $D e_{3}=\omega_{34} \otimes e_{4}$ and therefore $D \varepsilon=i \omega_{34} \otimes \varepsilon$. We now make two observations which are proved immediately after they are stated:

$$
\begin{gathered}
\left(\partial_{\bar{z}} \bar{\varepsilon}\right)^{T}=-\frac{1}{\left|F_{z}\right|^{2}}\left(\bar{\varepsilon} \cdot F_{\bar{z} \bar{z}}\right) F_{z}, \\
\left(\partial_{z} \bar{\varepsilon}\right)^{T}=-\frac{1}{\left|F_{z}\right|^{2}}\left(\bar{\varepsilon} \cdot F_{z z}\right) F_{\bar{z}}, \\
\partial_{z}\left(\frac{F_{z}}{\left|F_{z}\right|^{2}}\right)=\frac{\left(F_{z z}\right)^{N}}{\left|F_{z}\right|^{2}} .
\end{gathered}
$$

In the proof of (5.4) and (5.5) we will be using the Leibniz rule, the fact that $\varepsilon$ is a normal section and therefore that $\varepsilon \cdot F_{z}=\varepsilon \cdot F_{\bar{z}}=0$ and also the minimality of $F$, that is, $F_{z \bar{z}}=0$. We now compute

$$
\begin{aligned}
\left(\partial_{\bar{z}} \bar{\varepsilon}\right)^{T} & =\frac{1}{\left|F_{z}\right|^{2}}\left(\left(\partial_{\bar{z}} \bar{\varepsilon}\right) \cdot F_{z}\right) F_{\bar{z}}+\frac{1}{\left|F_{z}\right|^{2}}\left(\left(\partial_{\bar{z}} \bar{\varepsilon}\right) \cdot F_{\bar{z}}\right) F_{z} \\
& =-\frac{1}{\left|F_{z}\right|^{2}}\left(\bar{\varepsilon} \cdot F_{\bar{z} \bar{z}}\right) F_{z} .
\end{aligned}
$$

The proof of $\left(5.4^{\prime}\right)$ is identical to that of (5.4). We prove (5.5) by showing that $\left\{\partial_{z}\left(F_{z} /\left|F_{z}\right|^{2}\right)\right\}^{T}=0$. This is done by establishing that

$$
\left(\partial_{z}\left(\frac{F_{z}}{\left|F_{z}\right|^{2}}\right)\right) \cdot F_{z}=\left(\partial_{z}\left(\frac{F_{z}}{\left|F_{z}\right|^{2}}\right)\right) \cdot F_{\bar{z}}=0,
$$

both of which are easy and therefore left to the reader. Using (5.4) we may rewrite (5.3) as

$$
D_{\bar{z}} a^{1,0}=-\left(a \cdot \frac{F_{z}}{\left|F_{z}\right|^{2}}\right)\left(\bar{\varepsilon} \cdot F_{\bar{z} \bar{z}}\right) \varepsilon .
$$

From the above expression we compute $D_{z} D_{\bar{z}} a^{1,0}$ and on using (5.4 ) and (5.5) we obtain

$$
D_{z} D_{\bar{z}} a^{1,0}=-\left(a \cdot \frac{\left(F_{z z}\right)^{N}}{\left|F_{z}\right|^{2}}\right)\left(\bar{\varepsilon} \cdot F_{\bar{z} \bar{z}}\right) \varepsilon .
$$

(5.1) is now established by writing $\left(F_{z z}\right)^{N}=\left(F_{z z}\right)^{1,0}+\left(F_{z z}\right)^{0,1}$ and using expressions similar to (5.2) for $\left(F_{z z}\right)^{1,0},\left(F_{z z}\right)^{0,1}$ and $\left(F_{\bar{z} \bar{z}}\right)^{1,0}$. q.e.d. 
We now let $s=f \sigma$ in the stability inequality (2.2) where $f$ is a smooth real valued function with compact support and $\sigma$ is a complex valued normal section which need not have compact support. $f$ has to be real valued because of the following computation:

$$
\begin{aligned}
\int_{M} f^{2}\left|\left(\partial_{z} \sigma\right)^{T}\right|^{2} d x d y \leqslant & \int_{M}\left|f_{\bar{z}}\right|^{2}|\sigma|^{2} d x d y+\int_{M} f f_{\bar{z}}\left(D_{z} \bar{\sigma} \cdot \sigma\right) d x d y \\
& +\int_{M} f f_{z}\left(\bar{\sigma} \cdot D_{\bar{z}} \sigma\right) d x d y+\int_{M} f^{2}\left(D_{z} \bar{\sigma} \cdot D_{\bar{z}} \sigma\right) d x d y
\end{aligned}
$$

On rewriting $f f_{\bar{z}}$ as $1 / 2\left(f^{2}\right)_{\bar{z}}$ and $f f_{z}$ as $1 / 2\left(f^{2}\right)_{z}$ and integrating by parts the second and third terms of the right-hand side of (5.7), we obtain

$$
\begin{aligned}
\int_{M} f^{2}\left|\left(\partial_{z} \sigma\right)^{T}\right|^{2} d x d y \leqslant & \int_{M}\left|f_{\bar{z}}\right|^{2}|\sigma|^{2} d x d y \\
& -\int_{M} f^{2} \operatorname{Re}\left(\bar{\sigma} \cdot D_{z} D_{\bar{z}} \sigma\right) d x d y
\end{aligned}
$$

By a computation identical to that used in proving (5.4) we may show that

$$
\left|\left(\partial_{z} \sigma\right)^{T}\right|^{2}=\left|\sigma \cdot F_{z z}\right|^{2} /\left|F_{z}\right|^{2} \text {. }
$$

Let $\lambda=2\left|F_{z}\right|^{2}$. Then $d A$, the element of area on $M$, is locally given by $\lambda d x d y$. Moreover, since $f$ is real,

$$
|d f|^{2}=\frac{1}{\lambda}\left\{\left(f_{x}\right)^{2}+\left(f_{y}\right)^{2}\right\}=\frac{4}{\lambda}\left|f_{\bar{z}}\right|^{2} .
$$

Therefore (5.8) may be rewritten as:

$$
\begin{aligned}
2 \int_{M} f^{2} \frac{\left|\sigma \cdot F_{z z}\right|^{2}}{\left|F_{z}\right|^{4}} d A+2 \int_{M} \frac{f^{2}}{\left|F_{z}\right|^{2}} & \operatorname{Re}\left(\overline{\boldsymbol{\sigma}} \cdot D_{z} D_{\bar{z}} \sigma\right) d A \\
& \leqslant \int_{M}|d f|^{2}|\sigma|^{2} d A
\end{aligned}
$$

We can now give the

Proof of Theorem I. Fix a unit vector $a \in \mathbf{C}^{4}$ and let $\sigma=a^{1,0}$ in (5.9). Then, using (5.1), we obtain

$$
\int_{M} f^{2} q d A \leqslant \int_{M}|d f|^{2}\left|a^{1,0}\right|^{2} d A \leqslant \int_{M}|d f|^{2} d A
$$

the last inequality being true because $\left|a^{1,0}\right| \leqslant|a|=1$. In (5.10)

$$
q=-\frac{2}{\left|F_{z}\right|^{4}} \operatorname{Re}\left\{\left(F_{z z}\right)^{1,0} \cdot\left(\left(F_{\bar{z} \bar{z}}\right)^{1,0} \cdot \bar{a}\right) a\right\} .
$$


A theorem of D. Fischer-Colbrie and R. Schoen [12, Theorem 1, p. 201] says that, since (5.10) holds for all smooth functions $f$ with compact support, then there exists a smooth function $u>0$ satisfying the equation

$$
\Delta u+q u=0 \text {. }
$$

Following Fischer-Colbrie and Schoen we let $w=\log u$. Then $w$ satisfies the equation

$$
\Delta w=-q-|d w|^{2}
$$

Multiplying (5.13) by $f^{2}$, where $f$ is a smooth real valued function with compact support and integrating over $M$ we obtain

$$
2 \int_{M} f(d f \cdot d w) d A=\int_{M} f^{2} q d A+\int_{M} f^{2}|d w|^{2} d A,
$$

where we have integrated $\int_{M} f^{2} \Delta w d A$ by parts. Using the inequality $2|f(d f \cdot d w)| \leqslant \theta f^{2}|d w|^{2}+|d f|^{2} / \theta$ for any $\theta>0$ in (5.14) yields

$$
\frac{1}{\theta} \int_{M}|d f|^{2} d A \geqslant \int_{M} f^{2} q d A+(1-\theta) \int_{M} f^{2}|d w|^{2} d A .
$$

We now let $a$ run over an orthonormal basis $\left\{a_{1}, a_{2}, a_{3}, a_{4}\right\}$ of $\mathbf{C}^{4}$ and we let $q_{j}$ stand for the expression in (5.11) with $a=a_{j}$. The solution of (5.12) with $q=q_{j}$ is denoted by $u_{j}$ and $w_{j}=\log u_{j}$. Summing up over $j \in\{1,2,3,4\}$ the inequality (5.15) with $q=q_{j}$ and $w=w_{j}$ we obtain

$$
8 \int_{M}|d f|^{2} d A \geqslant \int_{M} f^{2}\left(\sum_{j} q_{j}\right) d A+1 / 2 \int_{M} f^{2}\left(\sum_{j}\left|d w_{j}\right|^{2}\right) d A,
$$

where we have set $\theta=1 / 2$. But from (5.11) we see that

$$
\sum_{j} q_{j}=-\frac{2}{\left|F_{z}\right|^{4}} \operatorname{Re}\left\{\left(F_{z z}\right)^{1,0} \cdot\left(F_{\bar{z} \bar{z}}\right)^{1,0}\right\}=0
$$

because $b \cdot c=0$ when $b$ and $c$ are of the same type. Therefore (5.16) becomes

$$
\int_{M}|d f|^{2} d A \geqslant \int_{M} f^{2} r d A
$$

where

$$
r=\frac{1}{16} \sum_{j}\left|d w_{j}\right|^{2}
$$

Since (5.17) holds for all smooth functions $f$ of compact support, the theorem of Fischer-Colbrie and Schoen gives us the existence of $v>0$ with $\Delta v+r v=0$. But since $r \geqslant 0, v$ is superharmonic and therefore the parabolicity of $M$ implies that $v$ is constant. It follows from the equation for $v$ that $r$ vanishes identically. 
From (5.18) we then deduce that $w_{j}$ is constant for each $j$ and therefore so is $u_{j}$. From (5.12) with $u=u_{j}$ and $q=q_{j}$ we conclude that $q_{j} \equiv 0$ for each $j$.

Now at an arbitrary point $p$ of $M$, either $\left(F_{z z}\right)^{N}(p)=0$ or else we can let $a_{1}=\left(F_{z z}\right)^{N}(p) /\left|\left(F_{z z}\right)^{N}(p)\right|$. In the latter case, $q_{1}=0$ implies, by (5.11), that $\left|\left(F_{z z}\right)^{1,0}(p)\right|\left|\left(F_{z z}\right)^{0,1}(p)\right|=0$. This last equality obviously still holds if $\left(F_{z z}\right)^{N}(p)=0$. Therefore, from Corollary 5.4, we deduce that at least one of $\left(F_{z z}\right)^{1,0}$ or $\left(F_{z z}\right)^{0,1}$ vanishes identically. We now note that we can change the notion of type $(1,0)$ to that of type $(0,1)$ simply by changing the complex structure on $N M$ to be rotation by $90^{\circ}$ clockwise as opposed to counterclockwise. Therefore, without loss of generality, we may assume that $\left(F_{z z}\right)^{0,1}$ vanishes identically. But then, if $s$ is a local nonzero section of $V$ (the subbundle of type $(1,0)$ of $\left.N_{\mathbf{C}} M\right)$, we have, by the Leibniz rule and minimality (see the proof of (5.4)), that

$$
\left(\partial_{z} s\right)^{T}=-\frac{s \cdot F_{z z}}{\left|F_{z}\right|^{2}} F_{\bar{z}}=0,
$$

where the last equality follows from $0=\left(F_{z z}\right)^{0,1}=\left(F_{z z} \cdot s\right) \bar{s} /|s|^{2}$. By the discussion following the proof of Theorem $A$ in $\$ 3$, the hypotheses for Theorem A are now all fulfilled and Theorem I is proved.

Professor R. Schoen has pointed out that the proof of Theorem I can be modified slightly to prove

Theorem I'. Let $F: M^{2} \rightarrow T^{4}$ be an isometric stable minimal immersion of a compact oriented surface $M$ without boundary into the flat torus $T^{4}=\mathbf{R}^{4} / \mathbf{Z}^{4}$. Then $F$ is holomorphic with respect to some orthogonal complex structure on $T^{4}$.

Outline of Proof. The constant vector field $a$ on $\mathbf{R}^{4}$ descends as a global parallel vector field on $T^{4}$ which we still denote by $a$. The normal bundle of $M$, being oriented and two-real dimensional, still has a complex structure defined on it, namely, rotation by $90^{\circ}$. Thus $a^{1,0}$ can be defined and formula (5.1) is still valid as long as by $F_{z}$ we mean $F_{*}\left(\partial_{z}\right)$ and by $\left(F_{z z}\right)^{N}$ we mean $B\left(\partial_{z}, \partial_{z}\right)$, where $B: T_{\mathbf{C}} M \otimes T_{\mathbf{C}} M \rightarrow N_{\mathbf{C}} M$ is the second fundamental form of $M$ (similarly for $F_{\bar{z}}$ and $\left.\left(F_{\bar{z} \bar{z}}\right)^{N}\right) ; F_{z z}$ of course means $\nabla_{F_{*}(\partial z)}\left(F_{*}\left(\partial_{z}\right)\right)$, where $\nabla$ is covariant differentiation in the tangent bundle of $T^{4}$ by means of the flat Levi-Civita (Riemannian) connection. Inequality (5.10) is therefore still valid for all smooth real valued functions $f$ on $M$. This means that the lowest eigenvalue $\lambda_{1}$ of $-\Delta-q$ on $M$ is nonnegative. Therefore, if $u>0$ is the eigenfunction of $-\Delta-q$ corresponding to $\lambda_{1}$, then $\Delta u+q u \leqslant 0$. Setting $w=\log u$ yields $\Delta w \leqslant-q-|d w|^{2}$ from which we may again derive (5.15) and therefore (5.16) and (5.17). Taking $f$ to be identically 1 in (5.17) yields $r \equiv 0$ and therefore $q_{j} \equiv 0$ for each $j \in\{1,2,3,4\}$. Therefore, as in the proof of 
Theorem I, we conclude that at least one of $\left(F_{z z}\right)^{1,0}$ and $\left(F_{z z}\right)^{0,1}$ must vanish identically. The remark and discussion following the proof of Theorem A finish the proof of Theorem $1^{\prime}$.

Theorem II. Let $F: M^{2} \rightarrow \mathbf{R}^{4}$ be an isometric stable minimal immersion of a complete oriented surface $M$ into Euclidean 4-space. Suppose that one of the projections of the Gauss map for $F$ onto the factors of $S^{2} \times S^{2}$ omits an open set. Then $F$ is holomorphic with respect to some orthogonal complex structure on $\mathbf{R}^{4}$.

Remarks. (i) The Gauss map for a holomorphic immersion $F: M \rightarrow \mathbf{C}^{2}$ has the property that one of its projections onto the factors of $S^{2} \times S^{2}$ is constant (see, for example, [14] or [19]).

(ii) There exist many examples (see, for instance, Hoffman, Osserman, Schoen [16, §4]) of minimal immersions of the unit disk (in $\mathbf{C}$ ) with a complete metric into $\mathbf{R}^{4}$ and whose Gauss map possesses the property stated in Theorem II. In these examples, the projection of the Gauss map which omits an open set from the relevant factor of $S^{2} \times S^{2}$ is nonconstant and therefore Theorem II and Remark (i) imply that all these examples are unstable.

(iii) If both projections of the Gauss map for a minimal immersion $F$ : $M \rightarrow \mathbf{R}^{4}$ omit an open set, then $F(M)$ is a plane, even without the assumption of stability on $F$. This was proved by Chern [8] and also by Osserman [23].

Proof of Theorem II. Throughout this proof we will be using the notation in $\S 4$. Suppose that $\omega_{1}$ omits the open set $\Omega$. Then for any fixed $\omega_{0} \in \Omega$, $\omega_{1} /\left(\omega_{1}-\omega_{0}\right)$ is bounded; here we are identifying $S^{2}$ with $\mathrm{C} \cup\{\infty\}$ via stereographic projection. Thus $M$ admits a bounded holomorphic function, which, by Remark (i), may be assumed to be nonconstant. If $M$ is planar, then, from the classification theory of Riemann surfaces, we know that $M$ admits a nonconstant harmonic function with finite Dirichlet integral (see, for example, [1, Theorem 7E, p. 208]). If $M$ is not planar, then (by definition) there exists a closed curve on $M$ which does not separate $M$, which, in turn, implies the existence of a nonzero real harmonic differential which is square integrable (see, for example, [27, Theorem 8-1 and the preceding discussion on page 208]). In either case, we obtain the existence of a nontrivial real harmonic differential $\alpha$ on $M$ which is square integrable. Therefore, $\beta=\alpha+i * \alpha$ is a square integrable holomorphic differential, where $*$ is the Hodge operator. Define $\sigma$ by

$$
\begin{aligned}
\sigma & =\frac{1}{\sqrt{2}}\left(\frac{1+\left|\omega_{1}\right|^{2}}{1+\left|\omega_{2}\right|^{2}}\right)^{1 / 2} \frac{\beta}{(\partial F \cdot P)} \frac{e_{3}-i e_{4}}{\left(\omega_{1}-\omega_{0}\right)^{2}} \\
& =\frac{\beta}{(\partial F \cdot P)\left(\omega_{1}-\omega_{0}\right)^{2}} s,
\end{aligned}
$$


where $\left(e_{3}-i e_{4}\right)$ and $s$ are defined by (4.3) and (4.7) respectively. We recall from (4.7) that $s$ is a meromorphic section of $V$ and therefore so is $\sigma$ because $\beta /(\partial F \cdot P)\left(\omega_{1}-\omega_{0}\right)^{2}$ is a meromorphic function. We claim that $\sigma$ is a square integrable section of $V$. To verify this claim, we compute: by (4.7) and (4.1),

$$
\begin{aligned}
|\sigma|^{2} & =\frac{|\beta|^{2}}{|\partial F \cdot P|^{2}} \frac{1}{\left|\omega_{1}-\omega_{0}\right|^{4}} \frac{1+\left|\omega_{1}\right|^{2}}{1+\left|\omega_{2}\right|^{2}} \\
& =|\beta|^{2}\left(\frac{1+\left|\omega_{1}\right|^{2}}{\left|\omega_{1}-\omega_{0}\right|^{2}}\right)^{2} \leqslant C|\beta|^{2},
\end{aligned}
$$

where $C$ is a constant depending only on the lower bound for $\left|\omega_{1}-\omega_{0}\right|$. Therefore $\sigma$ is square integrable because $\beta$ is. The above calculation is, strictly speaking, valid only when $F_{z} \cdot P \neq 0$, that is, when both $\omega_{1}$ and $\omega_{2}$ are finite. However, by making the change of variables $\zeta_{1}=1 / \omega_{1}$ or $\zeta_{2}=1 / \omega_{2}$ or both, it is a simple matter to check that the final inequality in (5.20) always holds.

We now let $s=f \sigma$ in the stability inequality (2.2) where $\sigma$ is given by (5.19) and $f$ is real valued and with compact support. Keeping in mind that $0=D_{\bar{z}} \sigma$ $=\left(\partial_{\bar{z}} \sigma\right)^{N},(2.2)$ becomes the following inequality:

$$
2 \int_{M} f^{2}\left|(\partial \sigma)^{T}\right|^{2} \leqslant \int_{M}|d f|^{2}|\sigma|^{2}
$$

For any $R>0$, let $f_{R}$ be a smooth function which is identically 1 on $B_{R}(p)$, the geodesic ball of radius $R$ with centre at $p$ in $M$, zero outside $B_{2 R}(p)$ and $\left|d f_{R}\right|<C^{\prime} / R$ everywhere on $M$, where $C^{\prime}$ is a constant independent of $R$. Letting $f=f_{R}$ in (5.21) and letting $R \rightarrow \infty$ we see that, since $\int_{M}|\sigma|^{2}<\infty$, we have to have $(\partial \sigma)^{T} \equiv 0$. But $\sigma$ is a section of $V$ with isolated zeros and therefore, by Corollary 5.4 and the discussion following the proof of Theorem A in $\S 3$, the hypotheses for Theorem A are now all satisfied. Theorem II is thus proved under the assumption that $\omega_{1}$ omits an open set. If $\omega_{2}$ omits an open set $\Omega$ and $\omega_{0} \in \Omega$, then we let

$$
\sigma=\frac{\beta}{(\partial F \cdot P)\left(\omega_{2}-\omega_{0}\right)^{2}} \frac{\bar{s}}{|s|^{2}},
$$

where $\bar{s} /|s|^{2}$ is given by (4.7). We again have $D_{\bar{z}} \sigma=0$ and that $\sigma$ is square integrable. Therefore, the same argument as above shows that $(\partial \sigma)^{T}=0$. But $\sigma$ is now a section of $\bar{V}$. As in the proof of Theorem I, we switch the rôles of $V$ and $\bar{V}$ simply by reversing the complex structure on $N M$. The proof of Theorem II is therefore complete.

Remark. The above proof runs on lines similar to the proof of Theorem 2 in [12]. There, D. Fischer-Colbrie and R. Schoen use the existence of real 
harmonic square integrable differentials on the unit disk in $\mathbf{C}$ to show that there do not exist positive solutions of the equation $\Delta u-a K u=0$ for $a \geqslant 1$, where $K$ is the Gauss curvature of a complete metric on the unit disk in $\mathbf{C}$.

Theorem II'. Let $F: M \rightarrow \mathbf{R}^{4}$ be an isometric stable minimal immersion of a complete simply connected oriented surface $M$. Suppose that one of the projections of the Gauss map for $F$ onto the factors of $S^{2} \times S^{2}$ omits a set of positive logarithmic capacity. Then $F$ is holomorphic with respect to some orthogonal complex structure on $\mathbf{R}^{4}$.

Proof of Theorem $I I^{\prime}$. From the proof of Theorem II, it is clear that all we have to do is show the existence of a square integrable holomorphic section of $V$ (or $\bar{V}$ ). Without loss of generality we assume that it is $\omega_{1}$ which omits a set $\Omega$ of positive logarithmic capacity and also that $\infty \in \Omega$. Then, by one of the characterizations of sets of positive logarithmic capacity (see, for example, [24, Lemma 8.6 on p. 70]), there exists a harmonic function $h$ on $\Omega^{c}=S^{2} \backslash \Omega$ such that $h\left(\omega_{1}\right) \geqslant \log \left(1+\left|\omega_{1}\right|^{2}\right)$ for all $\omega_{1} \in \Omega^{c}$. Since $\omega_{1}: M \rightarrow \Omega^{c}$ is holomorphic, $h \circ \omega_{1}$ is harmonic on $M$. But $M$ is simply connected and therefore $h \circ \omega_{1}=$ $\operatorname{Re} \phi$ for some holomorphic function $\phi$ on $M$. Moreover, $M$ has a global coordinate $z$ defined on it (indeed $M$ is conformally equivalent to the unit disk in C because $M$ is simply connected and $\omega_{1}$ is a holomorphic function on $M$ which omits more than 2 points of $S^{2}$ ). Thus we may define the required section $\sigma$ of $V$ by

$$
\sigma=\frac{s}{e^{\phi}\left(F_{z} \cdot P\right)},
$$

where $s$ is given by (4.7). We compute

$$
\begin{aligned}
|\boldsymbol{\sigma}|^{2} & =\frac{1}{\left(e^{h\left(\omega_{2}\right)}\right)^{2}\left|F_{z} \cdot P\right|^{2}} \frac{1+\left|\omega_{1}\right|^{2}}{1+\left|\omega_{2}\right|^{2}} \\
& \leqslant \frac{1}{\left|F_{z} \cdot P\right|^{2}\left(1+\left|\omega_{2}\right|^{2}\right)\left(1+\left|\omega_{1}\right|^{2}\right)} \\
& =\frac{2}{\lambda}=2|d x|^{2}
\end{aligned}
$$

where $\lambda=2\left|F_{z}\right|^{2}$ and $x=\operatorname{Re} z$. Since we are identifying $M$ with the unit disk, $\int_{M}|d x|^{2}=\pi$ and therefore $\sigma$ is square integrable. The rest of the proof now proceeds as that for Theorem II and we regard the proof of Theorem II' $^{\prime}$ as complete.

Remark. This proof is based on Osserman's proof in [22] of the theorem which says that the Gauss map of a complete nonplanar minimal surface in $\mathbf{R}^{3}$ cannot omit a set of positive logarithmic capacity. 
Theorem III. Let $F: M \rightarrow \mathbf{R}^{4}$ be an isometric stable minimal immersion of a complete oriented surface $M$. If the Gauss map for $F$ is at least $1 / 3$ degenerate (that is, there exists a nonzero fixed vector $A \in \mathbf{C}^{4}$ such that $|A \cdot A| \geqslant \frac{1}{3}|A|^{2}$ and $\left.A \cdot F_{z} \equiv 0\right)$ then the image of $F$ is a plane.

Proof. Throughout this proof we will be using the notation of the proof of Theorem I. We will be using the version of the stability inequality given by (5.9) with $\sigma=A^{1,0}-\overline{A^{1,0}}$. We make the following claims which are proved after the proof of Theorem III is completed:

Claim (i). $s=A^{1,0}$ is a nowhere vanishing holomorphic section of $V$.

Claim (ii). $\bar{\sigma} \cdot D_{z} D_{\bar{z}} \sigma=|s|^{2}\left(\log |s|^{2}\right)_{\bar{z} z}$.

Claim (iii). $\left|\sigma \cdot F_{z z}\right|^{2}=|s|^{2}\left|F_{z}\right|^{4}(-K)+\operatorname{Re}(A \cdot A)\left|\left(F_{z z}\right)^{1,0}\right|^{2}$, where $K$ is the Gauss curvature of $M$.

Claim (iv).

$$
\left|\left(\log |s|^{2}\right)_{\bar{z}}\right|^{2}=\frac{\left|\left(F_{z z}\right)^{1,0}\right|^{2}}{\left|F_{z}\right|^{2}|s|^{2}}\left(|A|^{2}-|s|^{2}-\frac{|A \cdot A|^{2}}{4|s|^{2}}\right) .
$$

Therefore, by Claims (ii) and (iii) and the fact that $|\sigma|^{2}=2|s|^{2},(5.9)$ becomes

$$
\begin{aligned}
& \int_{M} f^{2}|s|^{2}\left\{(-K)+\frac{\operatorname{Re}(A \cdot A)}{|s|^{2}} \frac{\left|\left(F_{z z}\right)^{1,0}\right|^{2}}{\left|F_{z}\right|^{4}}+\frac{1}{\left|F_{z}\right|^{2}}\left(\log |s|^{2}\right)_{\bar{z} z}\right\} \\
& \quad \leqslant \int_{M}|d f|^{2}|s|^{2} .
\end{aligned}
$$

Since $s$ vanishes nowhere (by Claim (i)) we can let $f=g /|s|$ in the above inequality and integrate by parts on the right to obtain

$$
\int_{M} g^{2}\{(-K)+q\} \leqslant \int_{M}|d g|^{2}
$$

where

$$
q=\frac{\operatorname{Re}(A \cdot A)}{|s|^{2}} \frac{\left|\left(F_{z z}\right)^{1,0}\right|^{2}}{\left|F_{z}\right|^{4}}+\frac{1}{\left|F_{z}\right|^{2}}\left(\log |s|^{2}\right)_{\bar{z} z}-\frac{2|s|_{\bar{z} z}}{|s|\left|F_{z}\right|^{2}} .
$$

But by Claim (iv),

$$
\left(\log |s|^{2}\right)_{\bar{z} z}-\frac{2|s|_{\bar{z} z}}{|s|}=-\frac{\left|\left(F_{z z}\right)^{1,0}\right|^{2}}{2\left|F_{z}\right|^{2}|s|^{2}}\left(|A|^{2}-|s|^{2}-\frac{|A \cdot A|^{2}}{4|s|^{2}}\right) .
$$


Moreover, we can choose $A$ so that $\operatorname{Re}(A \cdot A)=|A \cdot A|$. Therefore

$$
\begin{aligned}
q & =\frac{\left|\left(F_{z z}\right)^{1,0}\right|^{2}}{\left|F_{z}\right|^{4}|s|^{2}}\left\{|A \cdot A|-\frac{1}{2}|A|^{2}+\frac{1}{2}|s|^{2}+\frac{1}{8} \frac{|A \cdot A|^{2}}{|s|^{2}}\right\} \\
& \geqslant \frac{1}{2} \frac{\left|\left(F_{z z}\right)^{1,0}\right|^{2}}{\left|F_{z}\right|^{4}|s|^{4}}\left(|s|^{2}-\frac{1}{6}|A|^{2}\right)^{2},
\end{aligned}
$$

where we have used $|A \cdot A| \geqslant \frac{1}{3}|A|^{2}$ to obtain the last inequality. Since (5.22) holds for all real valued functions $g$ of compact support, the theorem of D. Fischer-Colbrie and R. Schoen [12, Theorem 1, p. 201] gives the existence of $u>0$ satisfying $\Delta u+\{(-K)+q\} u=0$. The lift of $u$ to the universal cover of $M$ satisfies the same equation as $u$. By (5.23), $q \geqslant 0$ and therefore another theorem of D. Fischer-Colbrie and R. Schoen [12, Theorem 2, pp. 203-205] shows that the unit disk in $\mathbf{C}$ cannot be the universal cover of $M$, which must therefore be the complex plane. But then the lift of $u$ would be a positive superharmonic function on $\mathbf{C}$ because $-K \geqslant 0$ for a minimal surface in Euclidean space. Therefore $u$ must be constant and it follows from the equation for $u$ that $q \equiv 0$ and $K \equiv 0$. $M$ must therefore be a plane.

Proof of Claim (i). $A \cdot F_{z} \equiv 0$ and therefore by (5.3) and (5.4)

$$
D_{\bar{z}} s=-\frac{1}{\left|F_{z}\right|^{2}}\left(A \cdot F_{z}\right)\left(\bar{\varepsilon} \cdot F_{\bar{z} \bar{z}}\right) \varepsilon=0,
$$

where $\varepsilon=\left(e_{3}-i e_{4}\right) / \sqrt{2}$ as in Lemma 5.2. Thus $s$ is holomorphic. Moreover,

$$
A=\frac{1}{\left|F_{z}\right|^{2}}\left(A \cdot F_{\bar{z}}\right) F_{z}+(A \cdot \bar{\varepsilon}) \varepsilon+(A \cdot \varepsilon) \bar{\varepsilon}
$$

Therefore

$$
A \cdot A=2(A \cdot \bar{\varepsilon})(A \cdot \varepsilon)
$$

Thus, $0<\frac{1}{3}|A|^{2} \leqslant|A \cdot A|=2|A \cdot \bar{\varepsilon}||A \cdot \varepsilon|$. In particular, $A \cdot \bar{\varepsilon}$ never vanishes, that is, $s$ never vanishes.

Proof of Claim (ii). Since $s$ is a holomorphic section of $V, D_{z} s=\left(\partial_{z} \log |s|^{2}\right) s$ (see the proof that $\bar{s} /|s|^{2}$ is a holomorphic section of $\bar{V}$ towards the end of $\S 4$ ). Therefore,

$$
\begin{gathered}
D_{z} D_{\bar{z}} \sigma=D_{z} D_{\bar{z}}(s-\bar{s})=-D_{z}\left(\partial_{\bar{z}} \log |s|^{2}\right) \bar{s}=-\left(\log |s|^{2}\right)_{\bar{z} z} \bar{s}, \\
\bar{\sigma} \cdot D_{z} D_{\bar{z}} \sigma=-(\bar{s}-s) \cdot\left(\log |s|^{2}\right)_{\bar{z} z} \bar{s}=|s|^{2}\left(\log |s|^{2}\right)_{\bar{z} z} .
\end{gathered}
$$


Proof of Claim (iii).

$$
\begin{aligned}
\left|\sigma \cdot F_{z z}\right|^{2} & =\left|(s-\bar{s}) \cdot F_{z z}\right|^{2} \\
& =\left|s \cdot F_{z z}\right|^{2}+\left|\bar{s} \cdot F_{z z}\right|^{2}-2 \operatorname{Re}\left(s \cdot F_{z z}\right)\left(s \cdot F_{\bar{z} \bar{z}}\right) \\
& =|s|^{2}\left|\left(F_{z z}\right)^{N}\right|^{2}-2 \operatorname{Re}(A \cdot \bar{\varepsilon})\left(\varepsilon \cdot F_{z z}\right)(A \cdot \bar{\varepsilon})\left(\varepsilon \cdot F_{\bar{z} \bar{z}}\right) .
\end{aligned}
$$

From the Gauss equation, it follows that $\left|\left(F_{z z}\right)^{N}\right|^{2}=\left|F_{z}\right|^{4}(-K)$. We also have that $A \cdot F_{z} \equiv 0$ and therefore that $A \cdot F_{z z} \equiv 0$. But

$$
F_{z z}=\left(F_{z z}\right)^{N}+\frac{1}{\left|F_{z}\right|^{2}}\left(F_{z z} \cdot F_{\bar{z}}\right) F_{z}
$$

and so, $A \cdot\left(F_{z z}\right)^{N} \equiv 0$, that is

$$
(A \cdot \bar{\varepsilon})\left(\varepsilon \cdot F_{z z}\right)+(A \cdot \varepsilon)\left(\bar{\varepsilon} \cdot F_{z z}\right) \equiv 0 .
$$

Claim (iii) is now proved by using (5.27), (5.25) and the Gauss equation in the last line of (5.26).

Proof of Claim (iv). We first note that $|s|^{2}=\bar{A} \cdot s$ and therefore, $\left(|s|^{2}\right)_{\bar{z}}=\bar{A}$ - $\partial_{\bar{z}} s=\bar{A} \cdot\left(\partial_{\bar{z}} s\right)^{T}$ because $\left(\partial_{\bar{z}} s\right)^{N}=D_{\bar{z}} s=0$ (from Claim (i)). Using the complex conjugate of $\left(5.4^{\prime}\right)$ in this expression for $\left(|s|^{2}\right)_{\bar{z}}$ we see that

$$
\left|\left(|s|^{2}\right)_{\bar{z}}\right|^{2}=\frac{1}{\left|F_{z}\right|^{4}}\left|A \cdot F_{\bar{z}}\right|^{2}\left|\bar{s} \cdot F_{z z}\right|^{2} \text {. }
$$

From (5.24) and (5.25) we also have

$$
|A|^{2}=\frac{1}{\left|F_{z}\right|^{2}}\left|A \cdot F_{\bar{z}}\right|^{2}+|s|^{2}+\frac{|A \cdot A|^{2}}{4|s|^{2}} .
$$

Claim (iv) now follows easily from (5.28) and (5.29). The proof of Theorem III is complete.

Theorem IV. Let $F: M^{2} \rightarrow \mathbf{R}^{n}$ be an isometric stable minimal immersion of a complete oriented surface $M$ of finite total curvature and genus zero into Euclidean $n$-space. Then $F(M)$ lies in an even dimensional affine subspace of $\mathbf{R}^{n}$ and is holomorphic with respect to some orthogonal complex structure on this even dimensional affine subspace.

Remark. Theorem IV is in some sense similar to the theorem of Calabi [5] about minimal immersions from $S^{2}$ into $S^{n}$, although the proof is quite different.

Before proving Theorem IV we prove the following

Lemma 5.3. Let $F: M \rightarrow \mathbf{R}^{n}$ be an isometric stable minimal immersion of a complete oriented parabolic surface $M$ into Euclidean $n$-space. If $\sigma$ is a bounded holomorphic section of $N_{\mathbf{C}} M$ (that is, $|\sigma| \leqslant C$ for some constant $C$ and $D_{\bar{z}} \sigma=0$ ) then $(\partial \sigma)^{T}=0$. 
Proof. Letting $s=f \sigma$ in the stability inequality (2.2), with $f$ a $C^{\infty}$ real valued function of compact support, yields

$$
\int_{M} f^{2}\left|(\partial \sigma)^{T}\right|^{2} \leqslant \frac{1}{2} \int_{M}|d f|^{2}|\sigma|^{2} \leqslant \frac{C^{2}}{2} \int_{M}|d f|^{2} .
$$

Since (5.30) holds for all $C^{\infty}$ real valued functions of compact support, the theorem of D. Fischer-Colbrie and R. Schoen [12, Theorem 1, p. 201] asserts the existence of $u>0$ satisfying

$$
\Delta u+\frac{2}{C^{2}}\left|(\partial \sigma)^{T}\right|^{2} u=0 .
$$

But then $u$ is a positive superharmonic function and therefore, since $M$ is parabolic, $u$ must be constant. The equation for $u$ then implies that $(\partial \sigma)^{T}=0$. q.e.d.

We now make some remarks about complete minimal surfaces of finite total curvature in Euclidean space. By a theorem of Chern and Osserman [9], such surfaces are conformally equivalent to a compact Riemann surface with finitely many punctures. Moreover, the Gauss map extends to the compactified surface as a holomorphic map.

We now recall that over $G_{2, n}$ we have the tautological 2-plane bundle $\gamma_{2, n}$ whose fibre over a point $\Pi$ consists of the vectors in $\mathbf{R}^{n}$ which lie in $\Pi$. We also have the $(n-2)$ plane bundle $\gamma_{2, n}^{\perp}$ which is the orthogonal complement of $\gamma_{2, n}$ in $G_{2, n} \times \mathbf{R}^{n}$. The tangent and normal bundles of a surface immersed in $\mathbf{R}^{n}$ are isomorphic (geometrically) to $G^{*}\left(\gamma_{2, n}\right)$ and $G^{*}\left(\gamma_{2, n}^{\perp}\right)$ respectively, where $G$ is the Gauss map of the immersion. Thus, in the case of a complete minimal surface $M$ of finite total curvature in $\mathbf{R}^{n}$, the tangent and normal bundles of $M$ extend (with all their geometric structure) to some vector bundles on the compactified surface $\hat{M}$; these vector bundles are, of course, $\hat{G}^{*}\left(\gamma_{2, n}\right)$ and $\hat{G}^{*}\left(\gamma_{2, n}^{\perp}\right)$ respectively, where $\hat{G}$ is the extension of $G$ to $\hat{M}$. We let $\eta=\hat{G}^{*}\left(\gamma_{2, n}^{\perp}\right)$ and $\eta_{\mathbf{C}}=\eta \otimes_{\mathbf{R}} \mathbf{C}$.

We now give the

Proof of Theorem IV. We first recall that $v \cdot w$ is the scalar product of $v, w \in \mathbf{C}^{n}$ which is complex linear in both arguments. However, whenever we say that a vector $v$ is orthogonal to a vector $w$, we mean that $v$ is orthogonal to $w$ in the standard Hermitian inner product on $\mathbf{C}^{n}$; thus $v$ is orthogonal to $w$ if and only if $v \cdot \bar{w}=0$.

Since the surface is assumed to be of genus zero, the compactified surface $\hat{M}$ must be the two-sphere $S^{2}$. Moreover, by the proposition of Koszul and Malgrange mentioned in $\S 4, \eta_{C}$ can be made into a holomorphic vector bundle. Therefore, by a theorem of Grothendieck [13], $\eta_{\mathbf{C}}$ splits as the direct sum of 
holomorphic line bundles $L_{1} \oplus \cdots \oplus L_{n-2}$. Let $L_{1}, \cdots, L_{p}$ be the positive line bundles, $L_{p+1}, \cdots, L_{r}$ be the topologically trivial ones and $L_{r+1}, \cdots, L_{n-2}$ be all negative. By the Riemann Roch theorem we know that each of $L_{1}, \cdots, L_{p}, L_{p+1}, \cdots, L_{r}$ admits a holomorphic section $s_{j}, j \in\{1, \cdots, r\}$. Simply by restricting the domain of $s_{j}$ to $M$, we obtain $r$ linearly independent bounded holomorphic sections of $N_{\mathrm{C}} M$. (Throughout this proof, linear independence of sections is meant over the continuous functions, not pointwise.) We know that $c_{1}\left(\eta_{\mathbf{C}}\right)=0$ because $\eta_{\mathbf{C}}$ is the complexification of a real vector bundle ( $c_{1}$ denotes first Chern class). But $c_{1}\left(\eta_{\mathbf{C}}\right)=c_{1}\left(L_{1}\right)+\cdots+c_{1}\left(L_{n-2}\right)$. Therefore only the following two cases arise.

Case (i). $L_{1}, \cdots, L_{n-2}$ are all trivial. In this case Lemma 5.3 says that $\left(\partial s_{j}\right)^{T}=0$ for all $j \in\{1, \cdots, n-2\}$. But the $s_{j}$ may be chosen to have no zeros and then $s_{1}, \cdots, s_{n-2}$ span $N_{\mathrm{C}} M$. Therefore $(\partial s)^{T}=0$ for all sections $s$ of $N_{\mathrm{C}} M$. Thus, for any section $s$ of $N_{\mathrm{C}} M,(d s)^{T}=(\partial s)^{T}+\overline{(\partial \bar{s}}^{T}=0$. This means that $M$ is totally geodesic and therefore a plane. In particular, Theorem IV holds.

Case (ii). $p>1$ and $r<n-2$. From now on we adopt the following range of indices:

$$
\begin{array}{ll}
1 \leqslant \mu, \nu \leqslant p, & r+1 \leqslant a \leqslant n-2, \\
1 \leqslant j, k \leqslant r, & p+1 \leqslant A \leqslant n-2 .
\end{array}
$$

We note that $s_{\mu} \cdot s_{j} \equiv 0$. The reason is that

$$
\partial_{\bar{z}}\left(s_{\mu} \cdot s_{j}\right)=\left(D_{\bar{z}} s_{\mu}\right) \cdot s_{j}+s_{\mu} \cdot\left(D_{\bar{z}} s_{j}\right)=0 .
$$

Therefore $s_{\mu} \cdot s_{j}$ is constant (holomorphic functions on compact manifolds are constant). But since $L_{\mu}$ is positive, $s_{\mu}$ must vanish somewhere and therefore $s_{\mu} \cdot s_{j} \equiv 0$ as claimed. This means that the $s_{\mu}$ lie in the space orthogonal to the span of $\left\{\bar{L}_{1}, \cdots, \bar{L}_{r}\right\}$ which is $n-2-r$ dimensional. Therefore $p \leqslant n-2-r$. We also know that each of $L_{r+1}, \cdots, L_{n-2}$ admits a meromorphic section $s_{a}$, $a \in\{r+1, \cdots, n-2\}$, which has only poles. Therefore $s_{a} \cdot s_{A}$ is a meromorphic function which either vanishes identically or else has only poles. The latter is impossible and therefore we conclude that the $s_{a}$ lie in the space orthogonal to the span of $\left\{\bar{L}_{p+1}, \cdots, \bar{L}_{n-2}\right\}$ which is $p$-dimensional. Therefore $n-2-r$ $\leqslant p$ and combining this with the previous reversed inequality we conclude that $p=n-2-r$ and that the space orthogonal to the span of $\left\{\bar{L}_{1}, \cdots, \bar{L}_{r}\right\}$ is spanned by $L_{1}, \cdots, L_{p}$.

We now claim that $\left(\partial_{z} s_{j} \cdot s_{k}\right) d z$ is a holomorphic differential. To prove this claim all we have to check is that $\partial_{z}\left(\partial_{z} s_{j} \cdot s_{k}\right)=0$. But

$$
\begin{aligned}
\partial_{\bar{z}}\left(\partial_{z} s_{j} \cdot s_{k}\right) & =\partial_{\bar{z}} \partial_{z} s_{j} \cdot s_{k}+\partial_{z} s_{j} \cdot \partial_{\bar{z}} s_{k} \\
& =\partial_{z}\left(\partial_{\bar{z}} s_{j} \cdot s_{k}\right)-\partial_{\bar{z}} s_{j} \cdot \partial_{z} s_{k}+\partial_{z} s_{j} \cdot \partial_{\bar{z}} s_{k} .
\end{aligned}
$$


Since the $s_{j}$ are holomorphic, $\partial_{\bar{z}} s_{j}$ has only a tangential component and, by Lemma 5.3, $\partial_{z} s_{j}$ has only a normal component. Therefore, all three terms in the last equality above are zero and our claim is proved. But by the Riemann-Roch theorem, there are no nonzero holomorphic differentials on $S^{2}$. Therefore $\partial_{z} s_{j} \cdot s_{k} \equiv 0$ for all $j, k$. From the argument in the previous paragraph we deduce that $\partial_{z} s_{j}$ lies in the span of $\left\{L_{1}, \cdots, L_{p}\right\}$.

Let $\xi=L_{1} \oplus \cdots \oplus L_{p} \oplus L_{p+1} \oplus \cdots \oplus L_{r} \oplus\left(T_{\mathbf{C}} M\right)^{1,0}$. (Recall that the fibres of $\left(T_{\mathbf{C}} M\right)^{1,0}$ are spanned by $F_{z}$.) We claim that $d: \Gamma(\xi) \rightarrow \Gamma\left(\xi \otimes T^{*} M\right)$, that is, $d$ preserves $\xi$. The above discussion shows that $d s_{j} \cdot s_{k}=0$ for all $j, k$. Therefore, all that remains to be checked is that $\left(d F_{z}\right) \cdot F_{z}=0$ and $\left(d F_{z}\right) \cdot s_{j}=$ 0 . The former is obvious and the latter follows from minimality, the Leibniz rule and the fact that $\left(\partial_{z} s_{j}\right)^{T}=0$ (from Lemma 5.3). The claim is proved.

A consequence of this claim is that $\xi_{q}$, the fibre of $\xi$ over the point $q$ in $M$, is a fixed subspace of $\mathbf{C}^{n}$ (that is, $\xi_{q}$ gives the same subspace of $\mathbf{C}^{n}$ for all $q$ in $M)$; here we are projecting the fibres of $F^{*}\left(T_{\mathbf{C}} \mathbf{R}^{n}\right)=M \times \mathbf{C}^{n}$ onto $\mathbf{C}^{n}$ in the obvious way. To see this, let $v$ be a vector in $\xi_{q_{0}}$ for some fixed $q_{0}$ in $M$. Let $v_{\xi}(q)$ and $v_{\xi}^{\perp}(q)$ be the orthogonal projections of $v$ onto $\xi_{q}$ and the orthogonal complement of $\xi_{q}$ respectively. Then $0=d v=d v_{\xi}+d v_{\xi}^{\perp}$. But since $d$ preserves $\xi$, it also preserves the orthogonal complement of $\xi$. Therefore $d v_{\xi}=d v_{\xi}^{\perp}$ $=0$. But $v_{\xi}^{\perp}\left(q_{0}\right)=0$ and therefore $v_{\xi}^{\perp}(q)=0$ for all $q$. Since $v$ was any vector in $\xi_{q_{0}}$, we conclude, as desired, that $\xi_{q}$ is the same subspace of $\mathbf{C}^{n}$ for all $q$. Thus $\xi=M \times \Lambda$ where $\Lambda$ is a subspace of $\mathbf{C}^{n}$ of dimension $r+1$.

Now let $T=\Lambda \cap \bar{\Lambda}$. Note that, since the space orthogonal to the span of $\left\{\bar{L}_{1}, \cdots, \bar{L}_{r}\right\}$ is spanned by $\left\{L_{1}, \cdots, L_{p}\right\}$, we have that $\operatorname{span}\{\Lambda, \bar{\Lambda}\}=\mathbf{C}^{n}$. But $\operatorname{dim} \operatorname{sp}\{\Lambda, \bar{\Lambda}\}=\operatorname{dim} \Lambda+\operatorname{dim} \bar{\Lambda}-\operatorname{dim} T$ and so, $n=2 r+2-t$, where $t=$ $\operatorname{dim} T$. Thus $t=r-p$ (since $p+r=n-2$ from above).

If $T$ is empty, $t=0, r=p$ and all we have to do to prove Theorem IV is to apply Theorem A with $E=\left(T_{\mathbf{C}} M\right)^{1,0}$ and $V=L_{1} \oplus \cdots \oplus L_{p}$. If $T$ is nonempty, then since $T$ is preserved by complex conjugation, $T=W \otimes_{\mathbf{R}} \mathbf{C}$, where $W$ is a subspace of $\mathbf{R}^{n}$ of real dimension $t$ equal to $r-p$. Now note that $\left(T_{\mathbf{C}} M\right)^{1,0}$ is not preserved by complex conjugation and so, $M \times W$ is a subbundle of $N M$ which is parallel in $F^{*}\left(T \mathbf{R}^{n}\right)$. This means that $F(M)$ lies in an affine subspace of $\mathbf{R}^{n}$ orthogonal to $W$; the dimension of this affine subspace is $n-t=2 p+2$. It also means that $N_{\mathbf{C}} M$ splits as the Whitney sum $N_{\mathrm{C}} M=M \times T \oplus(M \times T)^{\perp}$, where $(M \times T)^{\perp}$ is the orthogonal complement of $M \times T$ in $N_{\mathbf{C}} M$; $(M \times T)^{\perp}$ is the complexified normal bundle of $M$ when $M$ is viewed as a surface immersed in the affine subspace orthogonal to $W$. Now let $v$ be a vector in $T$. Since $v \in \Lambda, v=\sum_{k=1}^{r} a_{k} s_{k}+a F_{z}$. Therefore $v \cdot s_{\mu}=0$ for all $\mu \in\{1, \cdots, p\}$. Similarly, since $v \in \bar{\Lambda}, v \cdot \bar{s}_{\mu}=0$ for all 
$\mu \in\{1, \cdots, p\}$. Thus $T$ is orthogonal to the fibres of $L_{1} \oplus \cdots \oplus L_{p} \oplus \bar{L}_{1}$ $\oplus \cdots \oplus \bar{L}_{p}$ over any point $q$ in $M$, that is, $L_{1} \oplus \cdots \oplus L_{p} \oplus \bar{L}_{1} \oplus \cdots \oplus \bar{L}_{p} \subset$ $(M \times T)^{\perp}$. But the linear independence of $\left\{s_{1}, \cdots, s_{p}\right\}$ and the fact that $s_{\mu} \cdot s_{\nu}=0$ for all $\mu, \nu \in\{1, \cdots, p\}$ imply that $s_{1}, \cdots, s_{p}, \bar{s}_{1}, \cdots, \bar{s}_{p}$ are linearly independent. Moreover, $\operatorname{dim}(M \times T)^{\perp}=2 p$ and therefore

$$
L_{1} \oplus \cdots \oplus L_{p} \oplus \bar{L}_{1} \oplus \cdots \oplus \bar{L}_{p}=(M \times T)^{\perp} .
$$

Theorem IV is now proved by applying Theorem A with $E=\left(T_{\mathbf{C}} M\right)^{1,0}$ and $V=L_{1} \oplus \cdots \oplus L_{p}$.

\section{References}

[1] L. V. Ahlfors \& L. Sario, Riemann surfaces, Princeton University Press, Princeton, 1960.

[2] Ju. A. Aminov, On the problem of stability of a minimal surface in a Riemannian space of positive curvature, Soviet Math. Dokl. 16 (1975) 1278-1281.

[3] J. L. Barbosa \& M. doCarmo, On the size of a stable minimal surface in $\mathbf{R}^{3}$, Amer. J. Math. 98 (1976) 515-528.

[4] S. Bernstein, Sur un théorème de géométrie et ses applications aux equations aux dérivées partielles du type elliptique, Comm. Soc. Math. Kharkov 15 (1915-1917) 38-45.

[5] E. Calabi, Minimal immersions of surfaces in Euclidean spheres, J. Differential Geometry 1 (1967) $111-125$.

[6] M. doCarmo \& C. K. Peng, Stable minimal surfaces in $\mathbf{R}^{3}$ are planes, Bull. Amer. Math. Soc. (N.S.) 1 (1979) 903-906.

[7] S. Y. Cheng \& S. T. Yau, Differential equations on Riemannian manifolds and their geometric applications, Comm. Pure Appl. Math. 28 (1975) 333-354.

[8] S. S. Chern, Minimal surfaces in an Euclidean space of $N$ dimensions, Differential and Combinational Topology, A symposium in honor of Marston Morse, Princeton University Press, Princeton, 1965, 187-198.

[9] S. S. Chern \& R. Osserman, Complete minimal surfaces in Euclidean $n$-space, J. Analyse Math. 19 (1967) 15-34.

[10] S. S. Chern \& E. Spanier, A theorem on orientable surfaces in four-dimensional space, Comment Math. Helv. 25 (1951) 205-209.

[11] H. Federer, Some theorems on integral currents, Trans. Amer. Math. Soc. 117 (1965) 43-67.

[12] D. Fischer-Colbrie \& R. M. Schoen, The structure of complete stable minimal surfaces in 3-manifolds of non-negative scalar curvature, Comm. Pure Appl. Math. 33 (1980) 199-211.

[13] A. Grothendieck, Sur la classification des fibrées holomorphes sur la sphère de Riemann, Amer. J. Math. 79 (1957) 121-138.

[14] D. A. Hoffman \& R. Osserman, The geometry of the generalized Gauss map, Mem. Amer. Math. Soc. vol. 28, No. 236, 1980.

[15] The Gauss map of surfaces in $\mathbf{R}^{n}$, preprint.

[16] D. A. Hoffman, R. Osserman \& R. M. Schoen, On the Gauss map of complete surfaces of constant mean curvature in $\mathbf{R}^{3}$ and $\mathbf{R}^{4}$, Comment Math. Helv., to appear.

[17] J. L. Koszul \& B. Malgrange, Sur certaines fibrées complexes, Arch. Math. 9 (1958) 102-109.

[18] H. B. Lawson, Jr., Lectures on minimal submanifolds, Vol. I, Publish or Perish, Berkeley, 1980.

[19] M. J. Micallef, Stable minimal surfaces in Euclidean space, Ph.D. thesis, New York University, October 1982.

[20] F. Morgan, On the singular structure of two-dimensional area minimizing surfaces in $\mathbf{R}^{n}$, preprint. 
[21] R. Osserman, Proof of a conjecture of Nirenberg, Comm. Pure Appl. Math. 12 (1959) 229-232.

[22] __ On complete minimal surfaces, Arch. Rational Mech. Anal. 13 (1963) 392-404.

[23] _ Global properties of minimal surfaces in $E^{3}$ and $E^{n}$, Ann. of Math. (2) 80 (1964) $340-364$.

[24] _ A survey of minimal surfaces, Van Nostrand Reinhold, New York, 1969.

[25] R. M. Schoen, L. Simon \& S. T. Yau, Curvature estimates for minimal hypersurfaces, Acta Math. 134 (1975) 275-288.

[26] Y. T. Siu \& S. T. Yau, Compact Kähler manifolds of positive bisectional curvature, Invent. Math. 59 (1980) 189-204.

[27] G. Springer, Introduction to Riemann surfaces, Addison-Wesley, Reading, MA, 1957.

[28] A. N. Wang, Contributions to differential geometry, Ph.D. thesis, University of California, Berkeley, 1981.

[29] W. Wirtinger, Eine determinantenidentität und ihre anwendung auf analytische gebilde und Hermitesche massbestimmung, Monatsh. Math. Physik 44 (1936) 343-365.

[30] F. Xavier, The Gauss map of a complete non-flat minimal surface cannot omit 7 points on the sphere, Ann. of Math. 113 (1981) 211-214.

Mathematical SCIENCES Research Institute 